\title{
Pratiques culturales, valeurs d'usage et perception des paysans des espèces compagnes du cacaoyer dans des agroforêts traditionnelles au centre de la Côte d'Ivoire
}

Farming practices, utilization values, and Farmers' Perception of cocoa trees' companion species of in Traditional Agroforests, Centre of Côte d'Ivoire

Constant Yves Adou Yao, Kouassi Bruno Kpangui, Bi Tra Aimé Vroh et Djakalia Ouattara

\section{(2) OpenEdition}

Journals

Édition électronique

URL : http://journals.openedition.org/ethnoecologie/2474

DOI : $10.4000 /$ ethnoecologie.2474

ISSN : 2267-2419

Éditeur

Laboratoire Eco-anthropologie et Ethnobiologie

\section{Référence électronique}

Constant Yves Adou Yao, Kouassi Bruno Kpangui, Bi Tra Aimé Vroh et Djakalia Ouattara, « Pratiques culturales, valeurs d'usage et perception des paysans des espèces compagnes du cacaoyer dans des agroforêts traditionnelles au centre de la Côte d'Ivoire », Revue d'ethnoécologie [En ligne], 9 | 2016, mis en ligne le 01 juillet 2016, consulté le 10 décembre 2020. URL : http://journals.openedition.org/ ethnoecologie/2474; DOI : https://doi.org/10.4000/ethnoecologie.2474

Ce document a été généré automatiquement le 10 décembre 2020.

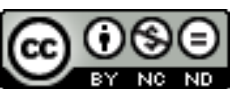

Revue d'ethnoécologie est mis à disposition selon les termes de la licence Creative Commons Attribution - Pas d'Utilisation Commerciale - Pas de Modification 4.0 International. 


\section{Pratiques culturales, valeurs d'usage et perception des paysans des espèces compagnes du cacaoyer dans des agroforêts traditionnelles au centre de la Côte d'Ivoire}

Farming practices, utilization values, and Farmers' Perception of cocoa trees' companion species of in Traditional Agroforests, Centre of Côte d'Ivoire

Constant Yves Adou Yao, Kouassi Bruno Kpangui, Bi Tra Aimé Vroh et Djakalia Ouattara

\section{Introduction}

1 Le déclin de la biodiversité se poursuit globalement et conduit à des changements majeurs dans les écosystèmes du monde et dans les sociétés humaines qui en dépendent (Cardinale et al. 2012). Depuis la première Stratégie Mondiale de la Conservation en 1980, la protection de la diversité biologique et la défense de la diversité culturelle se sont constituées en enjeu de société ces trente dernières années (Kasisi \& Jacobs 2002, Foyer 2008). En effet, les divers travaux réalisés indiquent que les systèmes de connaissances sociales, culturelles et environnementales des peuples autochtones et des communautés locales contribuent largement à la conservation de cette biodiversité et à sa gestion pour le bien-être des communautés (Takeuchi 2010, Berkes 2012, Hernandez-Morcillo et al. 2014).

2 La cacaoculture est considérée comme l'une des principales causes de dégradation du couvert forestier dans le monde tropical (Clough et al. 2011). Au-delà de son impact sur le couvert forestier, cette culture de rente est l'une des plus importantes, de par sa place dans l'économie mondiale, mais également, de par la diversité des services dont 
bénéficient les populations rurales (ICCO 2008). Ainsi, de nombreuses études se sont penchées sur la question des biens et services fournis par cette culture aux populations par la mise en avant de l'adoption de pratiques agroforestières (Sonwa et al. 2001, Jose 2009, Somarriba \& Beer 2011, Deheuvels et al. 2012, Cerda et al. 2014, Vaast \& Somarriba 2014). En effet, ces études ont montré que les systèmes agroforestiers traditionnels participeraient à la préservation de la diversité locale (Carrière 1999, Adou Yao \& N'Guessan 2006) et à la lutte contre la famine (Tano 2012). Par ailleurs, ces systèmes agroforestiers, selon leur niveau de complexité (floristique et/ou structurale), offrent une diversité d'habitats dont dépendent directement la culture principale, les cultures associées et les populations (Rice \& Greenberg 2000, Schroth et al. 2004). Dans ces mêmes études, il est fait mention des savoirs de communautés locales qui conduisent au maintien et à la préservation ainsi qu'à l'introduction de la diversité dans leurs plantations de cacaoyers (Adou Yao 2010, Kpangui et al. 2015).

En Côte d'Ivoire, principal pays producteur de cacao au monde, on estime à $66 \%$ les plantations cacaoyères sans ombrage sur l'ensemble du territoire (Ruf \& Zadi 1998). Cette situation est le résultat de la vulgarisation de variétés améliorées à haut rendement par les structures d'encadrement de paysans à la fin des années 1970. Cette dynamique d'extension de la cacaoculture va susciter un changement des pratiques culturales qui conduira au passage des systèmes agroforestiers pluristratifiés (complexes) à des cultures sous ombrage modéré ou plein soleil. Depuis quelques années, des auteurs (Tano 2012, Kpangui et al. 2015) soulignent le maintien et le retour des espèces végétales dans les plantations cacaoyères de l'ouest du pays, principale région productrice, dus à des pratiques et savoirs longtemps accumulés sur la culture du cacao.

Figure 1 : Agroforêt simplifiée dans la zone écologique de transition (contact forêt-savane) à Niamkey-Konankro, Centre de la Côte d'Ivoire

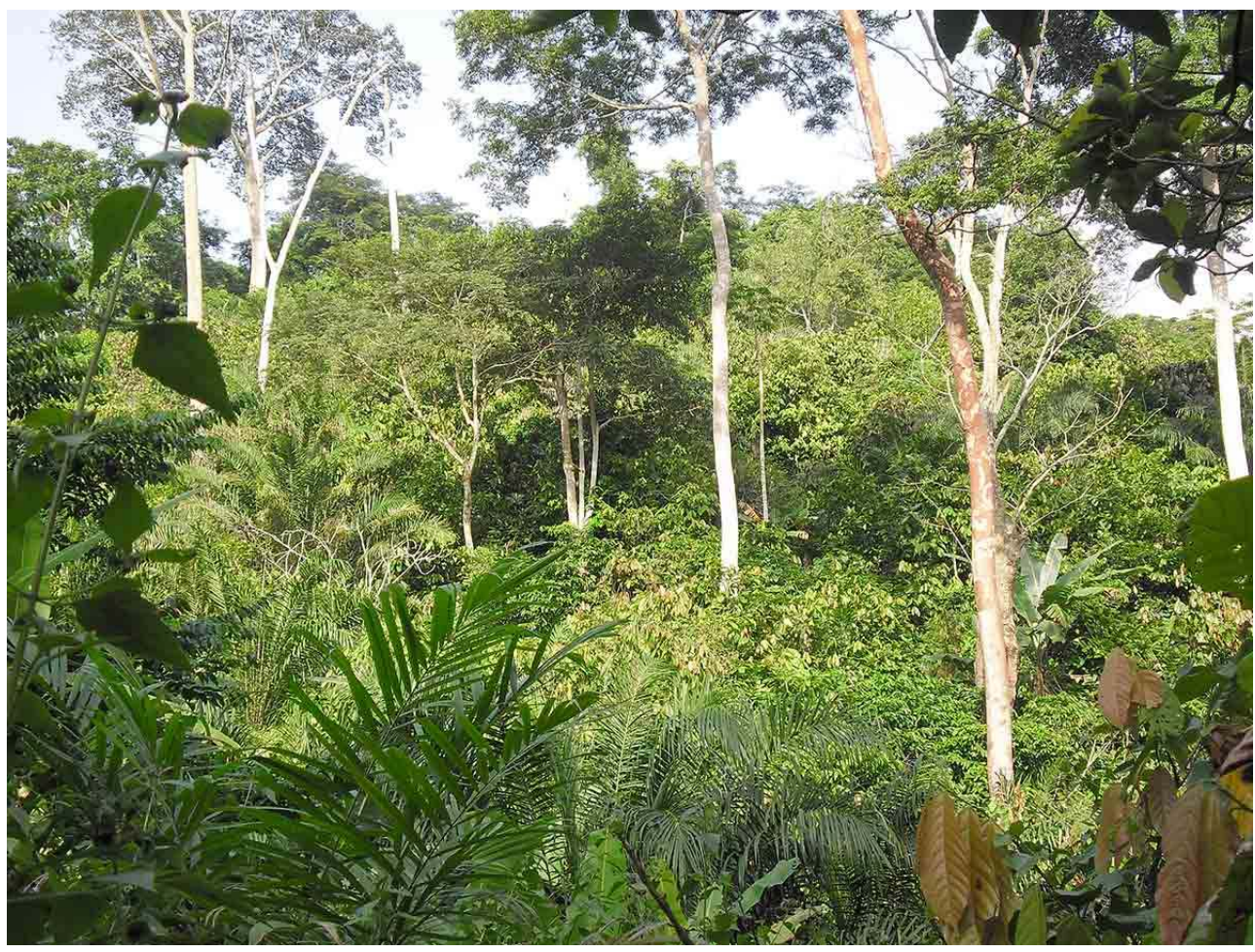

(c) Adou Yao 
Figure 2 : Agroforêt complexe à cacaoyers dans la zone écologique de transition (contact forêtsavane) à Niamkey-Konankro, Centre de la Côte d'Ivoire

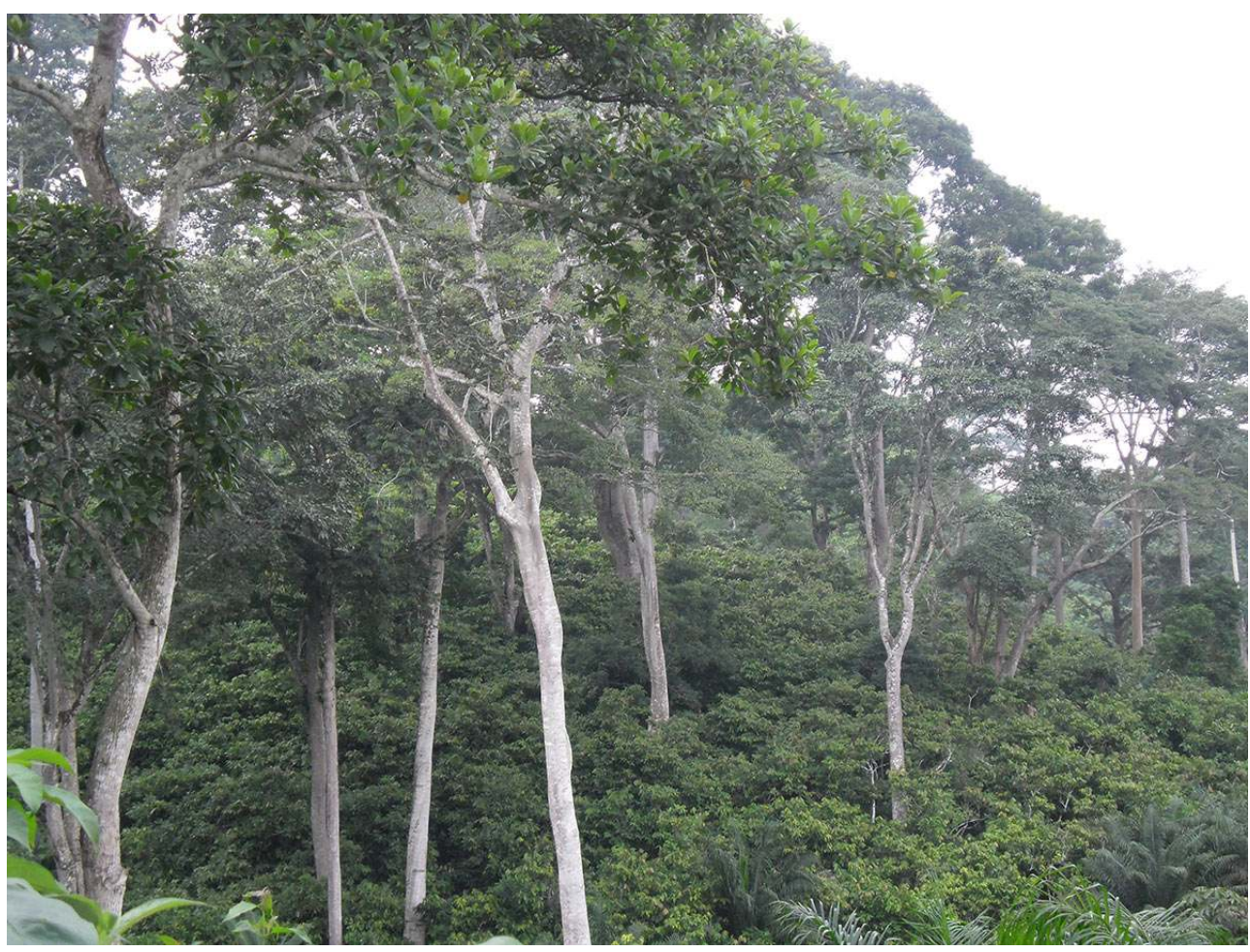

(c) Adou Yao

4 Dans les zones de contact forêt-savane considérées comme non favorables à la culture cacaoyère, la réussite des cultures de rentes a été attribuée à la présence de systèmes agroforestiers complexes dans lesquels des espèces locales et exotiques sont associées aux cacaoyers pour contourner les différentes contraintes du milieu (Herzog 1994). Aujourd'hui, malgré la disparition des forêts, le vieillissement des vergers, les changements de variétés et de pratiques, la culture cacaoyère persiste toujours dans cette région du pays. Dans un tel contexte, on peut s'interroger sur les pratiques mises en œuvre, les usages faits des espèces associées aux cacaoyers et sur les perceptions que les communautés locales ont des plantes qu'elles utilisent dans les systèmes agroforestiers traditionnels, dans une zone de transition forêt-savane peu adaptée à la cacaoculture. L'étude s'est donc fixé pour objectifs d'analyser les pratiques agroforestières traditionnelles de la sous-préfecture de Kokoumbo, les usages des espèces végétales présentes dans les plantations de la localité et les perceptions des populations sur les incidences des espèces compagnes sur le cacaoyer.

\section{Méthodes}

\section{Un site d'étude approprié à l'analyse des pratiques agroforestières}

5 L'étude a été conduite dans le centre de la Côte d'Ivoire, dans la sous-préfecture de Kokoumbo (Figure 3). 
Figure 3 : Localisation de la sous-préfecture de Kokoumbo au centre de la Côte d'Ivoire

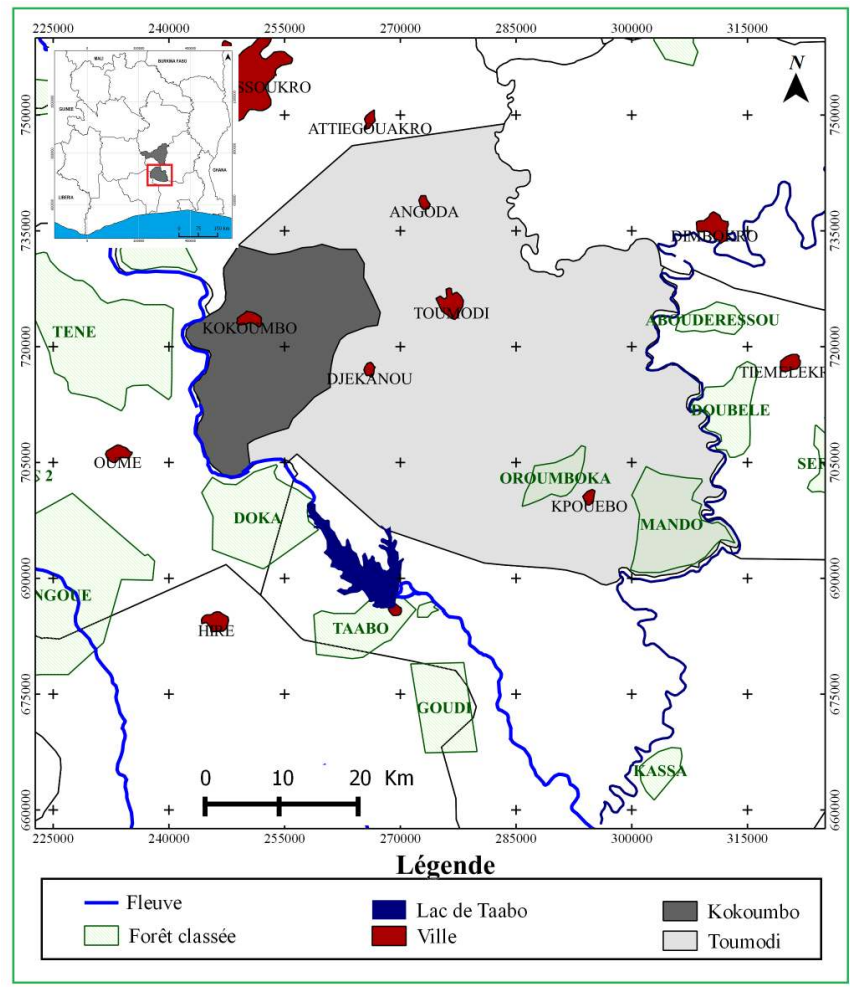

6 Les populations de la zone d'étude sont essentiellement représentées par le groupe ethnique Baoulé (autochtone), arrivé à la suite de diverses migrations à partir de la région de Bouaké. On y rencontre d'autres peuples (Malinké et Sénoufo du nord de la Côte d'Ivoire, Malien, etc.) venus essentiellement pour l'exploitation de l'or du KokoumboBoka (Chauveau 1979). L'intérêt de la zone d'étude réside dans le fait qu'elle est mitoyenne d'une des anciennes boucles du cacao (centre-ouest) en Côte d'Ivoire. On y rencontre également plusieurs types de systèmes agroforestiers (Kpangui et al. 2015). La particularité de la zone d'étude réside aussi dans sa végétation. C'est une zone de contact forêt-savane où les conditions climatiques et écologiques de production du cacao sont particulières : humidité faible, longs mois de saison sèche, formations végétales sur des flancs ou aux sommets des collines et fertilité relativement moindre (Yao-Kouamé 2007). Le climat, de type équatorial de transition, est caractérisé par deux saisons des pluies d'inégale importance, séparées par deux petites saisons sèches. Les précipitations annuelles varient entre 1106 et $1300 \mathrm{~mm}$ tandis que les températures annuelles varient entre $26,5^{\circ} \mathrm{C}$ et $28^{\circ} \mathrm{C}$. La végétation est constituée d'une mosaïque de savanes guinéennes et de forêts denses humides semi-décidues à Celtis spp. (Ulmaceae) et Triplochiton scleroxylon (Sterculiaceae) (Guillaumet \& Adjanohoun 1971). La transition entre ces deux types de formations végétales se fait sous la forme d'un gradient le long duquel la part des savanes est croissante depuis les blocs forestiers jusqu'à la région sub-soudanaise où les forêts ne représentent plus qu'une faible partie du territoire (Hiernaux 1975). Le sol de la région repose sur de vastes massifs granitiques, de roches métamorphiques et schisteuses (Perraud 1971). Les sols de la région sont relativement peu propices à la culture du cacao (Kpangui et al. 2015). Le relief de la zone, constitué de bas-plateaux et d'un système peu élevé de rides et de collines, appartient à « la chaîne baoulé ». Les altitudes varient entre 100 et $550 \mathrm{~m}$ avec pour principal sommet, le Kokoumbo-Boka (505 m). 


\section{Démarche méthodologique : une double approche ethnobotanique et phytoécologique}

7 La collecte des données de la présente étude s'est intéressée autant aux populations locales et leurs savoirs, savoir-faire, pratiques et connaissances qu'aux données biologiques. En effet, ce sont ces populations qui sont au cœur des interactions, des problèmes et des solutions à trouver (Roué et al. 2015).

Pour les connaissances locales en matière de gestion des espèces associées ainsi que leurs incidences sur les cacaoyers, des observations in situ ont été nécessaires. Trois sites ont été retenus en prenant en compte la prépondérance des systèmes agroforestiers à base de cacaoyers identifiés dans la zone étudiée par Kpangui et al. (2015) : Langossou, au contact des zones savanicoles; Niamkey-Konankro, en zone montagneuse et Kimoukro en zone forestière.

Dans ces trois villages, des questions ouvertes et des observations minutieuses et participatives de terrain auprès des petits exploitants agricoles ont permis de déterminer leurs perceptions et les raisons de la pratique de l'agroforesterie. Ces questions portaient sur le choix et les raisons de conservation ou d'introduction des espèces associées. Elles étaient destinées à savoir quels rôles ces espèces pourraient jouer dans leurs plantations : usages dans la vie quotidienne, effets sur la production de cacaoyers, sur la gestion de l'ombrage et sur la fertilité des sols? L'approche ethnobotanique qui a combiné des outils anthropologiques et ethnologiques, est essentielle pour une étude comme la nôtre qui met en face les connaissances locales et les savoirs scientifiques naturalistes (Nabanoga 2005). Pour parvenir à ces objectifs, nous avons été engagés dans le quotidien des activités des populations agricoles. Nous avons regardé ce que les agriculteurs faisaient et avons enregistré ce qu'ils disaient; et nous avons posé des questions sur le comportement des autres cultivateurs de cacaoyers qu'ils connaissent. Cette approche a permis de recenser avec tous les détails possibles les différentes pratiques de gestion des plantations agroforestières ainsi que d'analyser des usages des espèces associées par les agriculteurs locaux.

10 Pour l'analyse phytoécologique, nous avons mis en œuvre les outils classiques de ce type d'approche: inventaires botaniques de 105 placettes de $625 \mathrm{~m}^{2}(25 \mathrm{~m} \mathrm{x} 25 \mathrm{~m})$ dans certaines plantations choisies au hasard. Ces inventaires ont permis de dresser la liste d'espèces d'arbres dans les plantations, la liste des espèces énumérées par les populations comme plantes compagnes de cacaoyers et d'évaluer les services fournis mentionnés par les paysans. Il s'est agi principalement des services d'approvisionnement en produits (aliments, médicaments, bois de chauffage et autres utilisations) et des services d'appui (fertilité du sol et humidité, croissance des cacaoyers et production des cabosses). Nous avons effectué une analyse ethnographique combinant la transcription des textes d'entretiens et des observations participatives pour interpréter les idées et les raisons sous-jacentes avancées par les paysans: les pratiques culturales et les espèces associées aux cacaoyers, leur gestion et leurs rôles. Pour évaluer les relations existantes entre les différents usages des espèces et les perceptions de leur incidence dans la culture cacaoyère, une analyse factorielle de correspondance (AFC) couplée à une classification ascendante hiérarchique (CAH) a été effectuée grâce au package FactoMineR du logiciel R. 


\section{Résultats}

\section{Paysans et agroforêts traditionnelles à base de cacaoyers : des savoirs et savoir-faire hérités de longue date}

11 Nous avons interviewé 103 cultivateurs de cacao dans l'ensemble des trois villages de la sous-préfecture de Kokoumbo, avec un âge moyen de 44 ans. Quatre-vingts pourcent (80\%) parmi eux sont originaires de la région, $10 \%$ des nationaux ivoiriens venus d'autres régions et $10 \%$ des étrangers venus des pays ouest-africains. L'accès à la terre se faisait principalement par trois modes. Le premier mode est l'héritage ( $85 \%$ des cas) du père ou de la grande famille. Le don est le deuxième mode d'accès à la terre $(9,7 \%$ des cas). Ce don se fait à un individu, national ivoirien, qui a longtemps travaillé pour une famille qui le récompense en retour. Le dernier mode recensé est l'achat mais qui est très limité dans cette région où les populations sont en majorité des Akan qui ne vendent, en principe, jamais la terre. Même quand pour une raison ou une autre la vente doit se faire, c'est à un autre Akan qu'on va céder la terre des ancêtres. Les exploitations cacaoyères couvraient en moyenne 3 ha. Elles étaient âgées de 34 ans en moyenne, avec trois variétés principalement cultivées (Français: «Amelonado»; Ghana: «Foresteros HautAmazoniens et hybrides", et Mercedès: "Hybride Foresteros Haut-Amazoniens et mélange Amelonado et Trinitario »). La production annuelle moyenne est de 1106,56 kg/ paysan.

12 À la question "comment et pourquoi vous créez un champ de cacaoyers avec tant d'arbres à l'intérieur?» Le chef du village de Niamkey-Konankro, un vieil homme d'environ 70 ans répond :

«C'est mon grand-père et mon père qui nous l'ont enseigné. Et puis quand j'ai commencé à le faire de cette façon, j'ai vu que c'était bon ; mon champ hérité de mon père continue de produire, pas comme avant mais il produit encore après plus de 60 ans. J'ai même ajouté des orangers et avocatiers dedans pour gagner un peu d'argent encore ".

13 La mise en place d'une agroforêt à base de cacaoyers commence la plupart du temps par la coupe et le brûlis d'une végétation préexistante : forêt "naturelle », forêt dégradée, jachères ou ancienne plantation. Par la suite, on va planter une série de cultures vivrières. Dans celles-ci sont également laissés des arbres et arbustes que le paysan juge bien pour les cultures vivrières mais aussi pour les jeunes cacaoyers qu'il s'apprête à planter. Ainsi, à l'ombre des cultures et de bien d'autres arbres et arbustes maintenus, on sème directement les fèves ou les jeunes plants de cacaoyers issus de pépinières. Cependant, certains paysans coupent juste le sous-bois des forêts, les jachères ou les savanes guinéennes et sèment directement les fèves ou incorporent les jeunes plants issus des pépinières à l'ombre des arbres et des arbustes épargnés ou plantés dans les champs selon les connaissances et besoins du paysan. Les jeunes plants de cacaoyers sont donc intercalés avec plusieurs espèces telles que les avocatiers, les orangers, les bananiers, l'igname, le manioc, .... Les paysans préfèrent par-dessus tout, planter ou préserver, des arbres fruitiers, des plantes médicinales et des espèces qui ont une importance socioculturelle et/ou économique pour eux. À périodicité régulière, en général après la grande récolte, les paysans procèdent soit à l'abattage sélectif soit à l'ébranchage de certains arbres d'ombre selon les besoins. Toutes ces pratiques mises en œuvre par le paysan 
contribuent à la gestion de l'ombrage nécessaire à une bonne croissance et un bon développement des cacaoyers.

\section{Diversité des espèces compagnes du cacaoyer : favoriser les plantes à divers usages pour les populations}

Les enquêtes réalisées dans les plantations cacaoyères ont permis d'identifier 105 espèces couramment utilisées par les paysans. Ces espèces leur rendent des services d'approvisionnement. En considérant les différentes valeurs d'usages, les espèces utilisées comme bois de chauffage ( 56 espèces), médicinales (38 espèces) et alimentaires ( 28 espèces) sont les plus rencontrées dans les plantations. Les espèces utilisées comme bois d'œuvre et celles affectées à d'autres types d'usages sont les moins représentées avec six espèces (Figure 4).

Figure 4 : Histogrammes de distribution des espèces inventoriées suivant le type d'utilisation locale

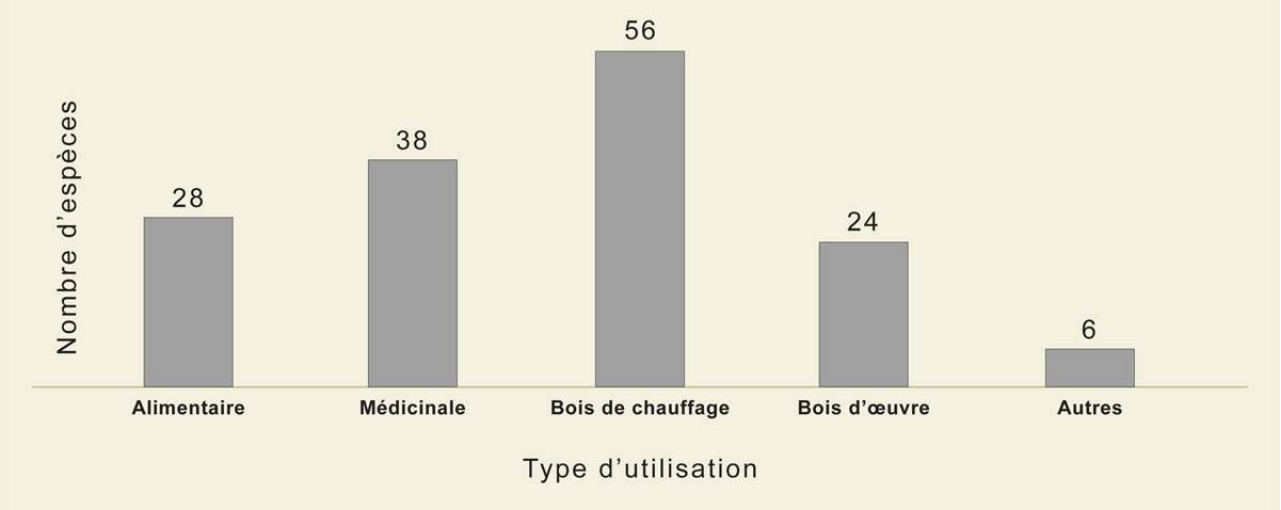

L'abondance des individus dans les plantations, varie suivant le type d'utilisation et la localité (Tableau 1). Les espèces alimentaires sont les plus conservées dans les plantations quelle que soit la localité. Les organes consommés sont principalement les fruits, les jeunes feuilles (préparation de sauce) et les graines (condiment, extraction d'huile) ainsi que la sève (vin de palme, de rônier). Les espèces alimentaires fréquemment rencontrées sont Musa paradisiaca (Musaceae), Persea americana (Lauraceae), pour les espèces exotiques et Elaeis guineensis (Arecaceae), Ricinodendron heudelotii (Euphorbiacéeé) et Cola nitida (Sterculiaceae) pour les espèces locales. Leurs proportions de tiges varient entre 65,02\% (Kimoukro) et 75,15 \% (Langossou).

Tableau 1 : Pourcentages des types d'utilisations des espèces de différentes localités

\begin{tabular}{|c|c|c|c|c|c|}
\hline Localités & Alimentaire & Chauffage & Medicinal & Bois d'œuvre & Autres usages \\
\hline Kimoukro & 65,02 & 26,78 & 13,81 & 7,77 & 8,97 \\
\hline Konankro & 73,60 & 24,61 & 18,42 & 10,18 & 19,49 \\
\hline Langossou & 75,15 & 19,95 & 8,88 & 3,39 & 16,22 \\
\hline Proportion générale & 72,84 & 23,30 & 14,36 & 7,42 & 16,74 \\
\hline
\end{tabular}

Les espèces servant de bois de chauffage et celles utilisées dans la médecine traditionnelle sont relativement bien représentées. Les espèces utilisées comme bois d'œuvre sont les moins conservées dans les plantations (Figure 5). La proportion moyenne de tiges est de $7,4 \%$, variant entre 3,4 \% (Langossou) et 10,2\% (Niamkey-Konankro). 
Parmi les espèces auxquelles les populations locales baoulé reconnaissent des usages, 92 sont issues de la flore locale ( 68 sont forestières, 24 espèces de transition forêt-savane) et 13 sont des espèces exotiques (Tableau 2). Les espèces locales forestières sont utilisées en majorité comme bois de chauffage $(56,2 \%)$ et plantes alimentaires (38,9\%). Seules 5,9\% d'entre elles sont utilisées comme bois d'œuvre. Les espèces exotiques, sont essentiellement alimentaires (99,9\%).

Tableau 2 : Variation des types chorologiques des espèces utilisées par les populations

\begin{tabular}{|c|l|c|c|c|c|c|c|}
\hline \multicolumn{1}{c|}{ Variables } & \multicolumn{1}{|c|}{ Type chorologique } & Alimentaire & Bois de chauffage & Médicinale & Bois d'œuvre & Autres & Total \\
\hline \multirow{2}{*}{ Richesse floristique } & Espèce locale forestière & 11 & 41 & 25 & 19 & 6 & 68 \\
\cline { 2 - 8 } & Espèce locale de transition & 5 & 12 & 12 & 4 & 0 & 24 \\
\cline { 2 - 9 } & Espèce exotique & 12 & 3 & 1 & 1 & 0 & 13 \\
\hline \multirow{2}{*}{ Abondance d'individus } & Espèce locale forestière (\%) & 38,9 & 56,2 & 17,2 & 5,9 & 28,3 & 29,3 \\
\cline { 2 - 9 } & Espèce locale de transition (\%) & 28,6 & 51,2 & 48,8 & 29,8 & 0,0 & 31,7 \\
\hline & Espèce exotique (\%) & 99,9 & 1,7 & 1,8 & 0,1 & 0,0 & 20,7 \\
\hline
\end{tabular}

Quant aux espèces locales de la zone phytogéographique de transition forêt-savane, elles sont principalement utilisées comme bois de chauffage $(51,2 \%)$ et plantes médicinales $(48,8 \%)$. Elles sont à l'opposé, moins représentées chez les plantes alimentaires (28,6\%).

\section{Perception du rôle des espèces associées aux cacaoyers : des plantes à diverses incidences sur le sol et le cacaoyer}

Outre les avantages en termes d'approvisionnement ci-dessus mentionnés, 65 espèces végétales ont été citées comme pouvant avoir des incidences sur le sol (fertilité, humidité, assèchement), sur le développement des cacaoyers (compétition avec le cacaoyer, des hôtes de bio-agresseurs et hôte des Loranthaceae) et sur la production de cabosses (accroissement de la production des cabosses, baisse de la floraison). Parmi ces espèces, 56 sont citées à Niamkey-Konankro, 51 à Langossou et 33 à Kimoukro. Il s'agit pour les plus citées, de Ceiba pentandra (Bombacaceae) et de Milicia excelsa (Moraceae) avec des fréquences respectives de $47,6 \%$ et $46,6 \%$, de Persea americana (Lauraceae) et Citrus sinensis (Rutaceae) avec des proportions respectives de $39,8 \%$ et $36,9 \%$.

Une ordination couplée à une classification ascendante hiérarchique réalisée à partir de neuf critères décrivant la perception des paysans, les différents usages et les localités d'enquêtes, a permis de distinguer cinq groupes d'espèces (Figures 5 et 6 ). 
Figure 5 : Diagramme d'ordination des espèces arborescentes associées aux cacaoyers

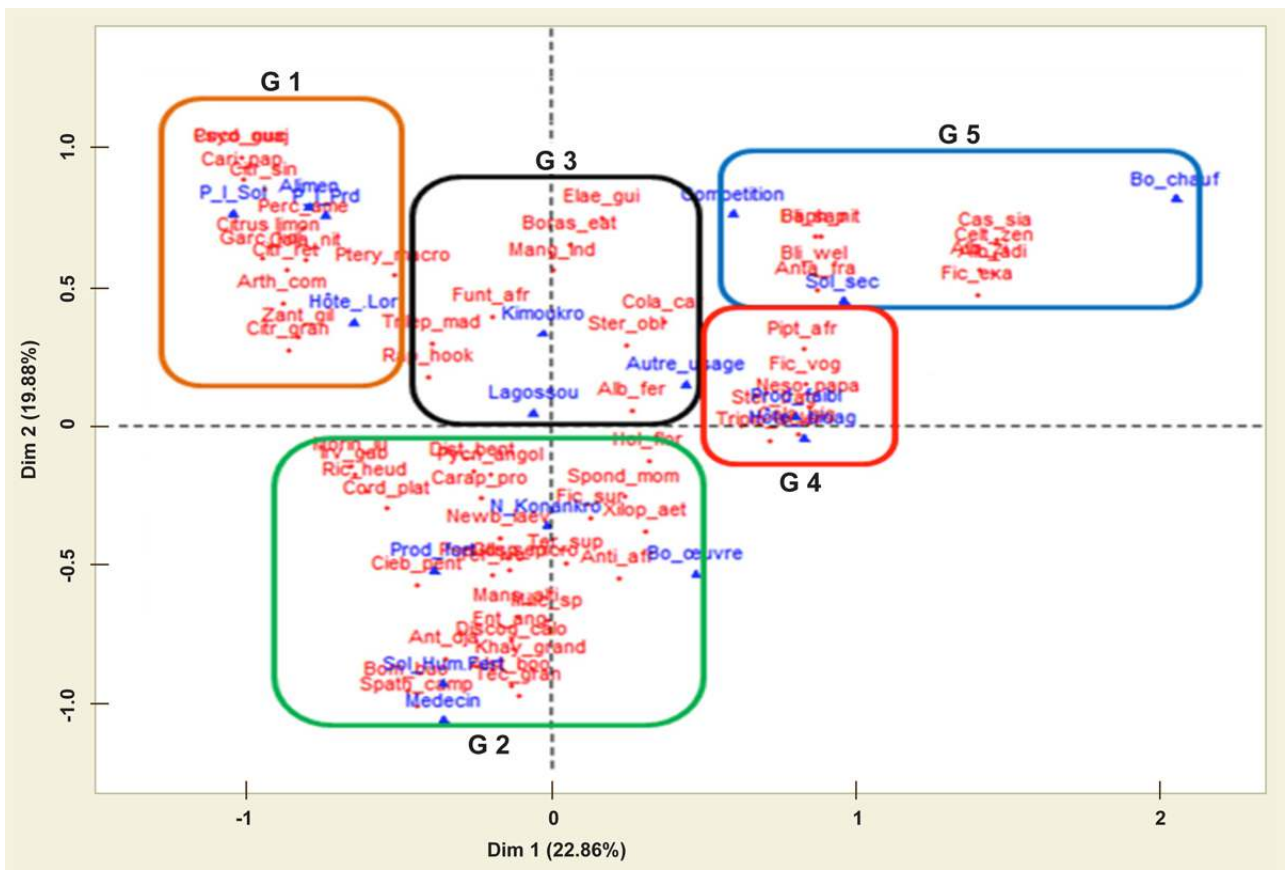

G1 : espèces reconnues par les paysans comme n'ayant aucune incidence sur la production ; G2 : espèces reconnues comme accroissant la fertilité des sols et la production ; G3 : espèces pour lesquelles les avis ne sont pas tranchés sur leurs incidences ; G4 : espèces vues comme asséchant les sols ; G5 : espèces à influence négative sur le développement des cacaoyers

Figure 6 : Répartition des différents groupes d'espèces identifiés à partir de la classification ascendante hiérarchique (CAH)

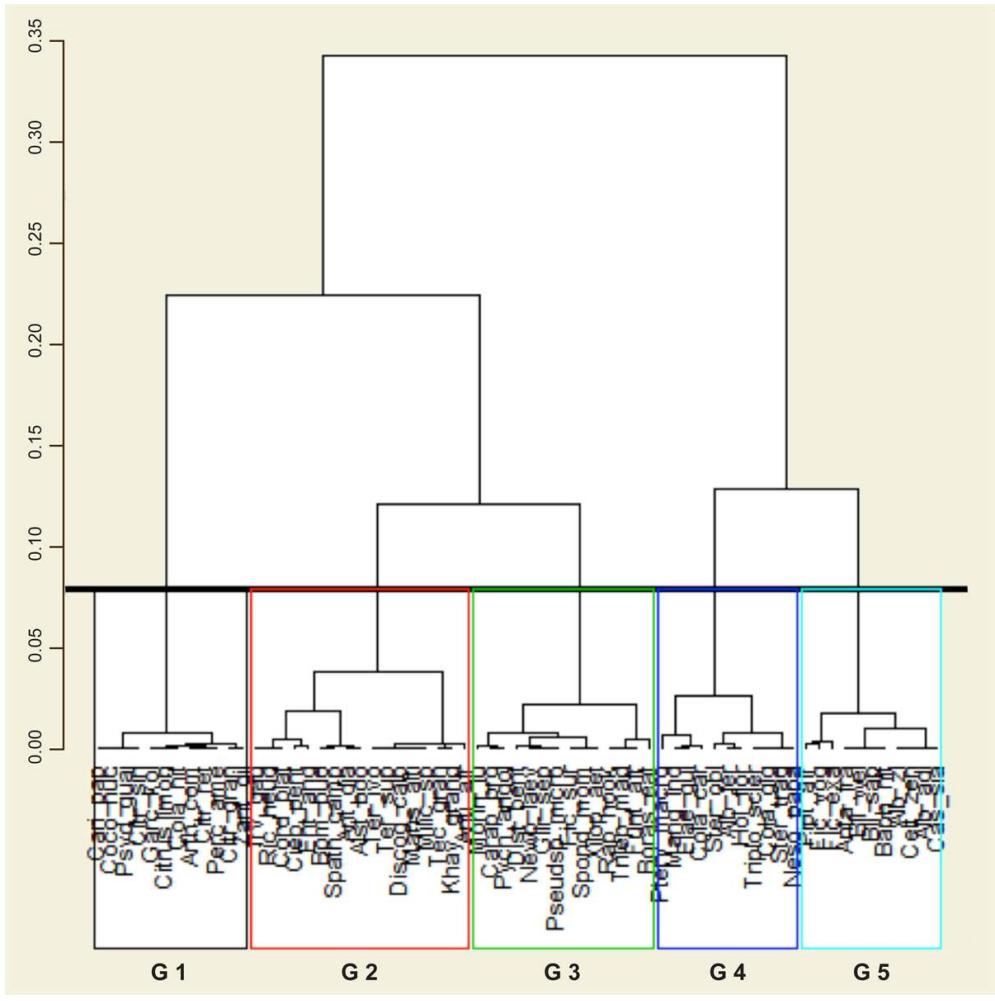




\begin{tabular}{|c|c|c|c|c|c|}
\hline Code_spc & Espèces & Cluster & Code_spc & Espèces & Cluster \\
\hline Arth_com & Arthocarpus communis & G1 & Alst_boo & Alstonia boonei & G2 \\
\hline Carap_pro & Carapa procera & G1 & Ant_dja & Anthocleista djalonensis & G2 \\
\hline Cari_pap & Carica papaya & G1 & Anti_afr & Antiaris africana & G2 \\
\hline Citr_gran & Citrus grandis & G1 & Bom_buo & Bombax buonopozense & G2 \\
\hline Citr_ret & Citrus reticulata & G1 & Cieb_pent & Ceiba pentandra & G2 \\
\hline Cit__sin & Citrus sinensis & G1 & Cola_gig & Cola gigantea & G2 \\
\hline Citrus_lim & Citrus limon & G1 & Discog_calo & Discoglypremna caloneura & G2 \\
\hline Coco_nuc & Cocus nucifera & G1 & Ent_ang & Entandrophragma angolense & G2 \\
\hline Cola_nit & Cola nitida & G1 & Fic_sur & Ficus sur & G2 \\
\hline Cord_plat & Cordia platythyrsa & G1 & Hol_flor & Holarrhena floribunda & G2 \\
\hline Garc_kol & Garcinia kola & G1 & Irv_gab & Invingia gabonensis & G2 \\
\hline Morin_lu & Morinda lucida & G1 & Khay_grand & Khaya grandifoliola & G2 \\
\hline Perc_ame & Persea americana & G1 & Mang_ind & Mangifera indica & G2 \\
\hline Psyd_guaj & Psydium guajava & G1 & Mans_alti & Mansonia altissima & G2 \\
\hline Ptery_macro & Pterygota macrocarpa & G1 & Milic_sp & Milicia sp & G2 \\
\hline Alb_fer & Albizia ferruginea & G3 & Pycn_angol & Pycnanthus angolensis & G2 \\
\hline Boras_eat & Borassus aethiopum & G3 & Ric_heud & Ricinodendron heudelotii & G2 \\
\hline Cola_car & Cola caricaefollia & G3 & Spath_camp & Spathodea companulata & G2 \\
\hline Elae_gui & Elaeis guineensis & G3 & Spond_mom & Spondias mombin & G2 \\
\hline Funt_afr & Funtumia africana & G3 & Ster_trag & Sterculia tragacantha & G2 \\
\hline Rap_hook & Raphia hookeri & G3 & Tec_gran & Tectona grandis & G2 \\
\hline Ster_obl & Sterculia oblongum & G3 & Ter_ivo & Terminalia ivorensis & G2 \\
\hline Trilep_mad & Trileplisium madagascariensis & G3 & Ter_sup & Terminalia superba & G2 \\
\hline Alb_adi & Albizia adianthifolia & G5 & Xilop_aet & Xylopia aethipica & G2 \\
\hline Alb_zi & Albizia zigia & G5 & Dist_bent & Distemonanthus benthamianus & G4 \\
\hline Anta_fra & Anthonotha fragans & G5 & Fic_vog & Ficus vogeliana & G4 \\
\hline Baph_nit & Baphia nitida & G5 & Glir_sep & Gliricidia sepium & G4 \\
\hline Bli_sap & Blighia sapida & G5 & Neso_papa & Nesogordonia papaverifera & G4 \\
\hline Bli_wel & Blighia welwitchii & G5 & Newb_laev & Newbouldia laevis & G4 \\
\hline Cas_sia & Cassia siamea & G5 & Pipt_afr & Piptadeniastrum africanum & G4 \\
\hline Celt_zen & Celtis zenkeri & G5 & Pseudsp_micro & Pseudospondias macrocarpum & G4 \\
\hline Fic_exa & Ficus exasperata & G5 & Triplo_scler & Triplochiton scleroxylon & G4 \\
\hline
\end{tabular}

21 Le groupe G1 est constitué de 13 espèces considérées par les paysans comme n'ayant aucune incidence ni sur la production et le développement des cacaoyers, ni sur le sol. Elles sont principalement conservées pour l'alimentation des populations. Les espèces les plus citées et observées dans les parcelles de cette classe sont : Cola nitida, Persea americana et Citrus sinensis (Tableau 3).

Tableau 3 : Espèces caractéristiques des différents groupes selon les perceptions des populations

\begin{tabular}{|c|c|c|c|}
\hline Groupe d'espèces & Nom scientifique & Proportion de paysans ayant citée & Fréquence d'observation dans les parcelles \\
\hline \multirow{3}{*}{ Groupe G1 } & Persea americana & 39,8 & 47,62 \\
\hline & Citrus sinensis & 36,89 & 15,24 \\
\hline & Cola nitida & 24,27 & 26,67 \\
\hline \multirow{3}{*}{ Groupe G2 } & Ceiba pentandra & 47,57 & 9,52 \\
\hline & Milicia regia & 46,6 & 10,48 \\
\hline & Antiaris africana & 19,41 & 11,43 \\
\hline \multirow{3}{*}{ Groupe G3 } & Alstonia boonei & 35,92 & 15,24 \\
\hline & Pycnanthus angolensis & 6,79 & 7,71 \\
\hline & Holarrhena floribunda & 5,83 & 0,95 \\
\hline \multirow{3}{*}{ Groupe G4 } & Elaeis guineensis & 19,41 & 59,05 \\
\hline & Triplochiton scleroxylon & 25,24 & 1,90 \\
\hline & Cola gigantea & 22,33 & 1,90 \\
\hline \multirow{3}{*}{ Groupe G5 } & Albizia zygia & 19,41 & 3,81 \\
\hline & Celtis zenkeri & 5,83 & 4,76 \\
\hline & Ficus exasperata & 14,56 & 5,71 \\
\hline
\end{tabular}

22 La seconde catégorie d'espèces (groupe G2) se rencontre principalement dans la zone de Niamkey-Konankro. Quinze espèces sont perçues par les paysans comme pouvant améliorer la fertilité des sols et accroître la production des cacaoyers. Elles sont principalement utilisées comme bois d'œuvre et en médicine traditionnelle. Trois espèces sont les plus citées et rencontrées dans les plantations : Ceiba pentandra, Milicia excelsa et Antiaris africana. 
23 Le troisième groupe G3 rassemble 16 espèces dont la majorité est très peu citée ou rencontrées dans l'ensemble des localités. Il s'agit d'espèces pour lesquelles les avis restent partagés dans la population interrogée quant à leur possible incidence sur le sol ou la production des cacaoyers. Les plus caractéristiques de ce groupe sont Alstonia boonei et Pycnanthus angolensis, perçues comme pouvant favoriser la production des cacaoyers mais aussi ce sont les principaux hôtes de bio-agresseurs (écureuils et mirides).

Les deux dernières catégories d'espèces (G4 et G5) sont considérées comme non favorables à la culture cacaoyère. Le groupe G4 regroupe 10 espèces associées à une faible production due soit à l'assèchement du sol pendant la saison sèche, soit au rôle en tant qu'hôte des bio-agresseurs. Leur maintien dans les plantations est lié à diverses utilisations faites par les populations (Figures 5 \& 6). Les espèces les plus citées sont: Nesogordonia papaverifera dont les racines jugées superficielles retardent la croissance des jeunes plants; Elaeis guineensis dont la forte densité dans les plantations pourrait entraîner la mort des cacaoyers ; Cola gigantea pour son ombrage excessif. Le groupe G5 rassemble 13 espèces qui influenceraient négativement le développement des cacaoyers. Leur maintien est lié à leur utilisation comme bois de chauffage. On peut citer Albizia zygia ,Celtis zenkeri et Ficus exasperata.

\section{Discussion}

\section{Utilisation des espèces par les populations}

L'agroforesterie permet de combler différents besoins des populations rurales : bois de chauffage, aliments nutritifs et remèdes provenant de plantes médicinales. Il s'agit en plus de la production cacaoyère, de nombreux biens et services nécessaires aux populations rurales (Herzog 1994, Sonwa et al. 2001). Les résultats obtenus dans la présente étude montrent, en effet, que les espèces préservées dans les plantations sont utilisées à diverses fins en plus de leur fonction principale qui est de servir d'ombrage aux cacaoyers. L'utilisation principale des espèces est le bois de chauffage. Les usages médicinaux, le bois d'œuvre et les plantes alimentaires sont, à un degré moindre, des usages accordés également à ces espèces compagnes. Cette situation, similaire à celle observée par Herzog (1994) dans des localités voisines, pourrait s'expliquer par le fait que dans les villages, le bois constitue la principale source d'énergie des foyers. Il constitue également une importante source de revenus pour les jeunes qui font de sa collecte une activité principale. En effet, les espèces utilisées pour l'énergie domestique étaient, autrefois, principalement récoltées dans les forêts. Ces dernières étant de plus en plus rares et éloignées des villages, la récolte de bois de chauffage se fait préférentiellement dans les plantations cacaoyères évitant aux femmes de parcourir de grandes distances. La raréfaction du bois de chauffage dans les cacaoyères, les obligerait donc à en acheter auprès des jeunes qui les récoltent dans les forêts restantes. L'abondance des espèces à usages médicinaux témoigne d'une bonne connaissance de leurs vertus par les paysans. Leur importance dans les plantations pourrait se justifier par le fait que face à la pauvreté, les paysans se tournent vers la médecine traditionnelle pour couvrir leurs besoins en matière de santé. En effet, les structures conventionnelles (centre de santé) demeurent relativement chères par rapport à leur pouvoir d'achat et rares dans les différentes localités. Les populations prélèvent ainsi dans leurs champs, des plantes médicinales connues pour soulager certaines affections. C'est le cas d'Alstonia boonei 
(Apocynaceae) connue sous le nom local de Emien qui est reconnue pour son efficacité dans le traitement du paludisme (Vroh et al. 2014). Les plantes à usage alimentaire occupent le troisième rang des services d'approvisionnement fournis par les espèces compagnes des cacaoyers, aux populations de la zone d'étude. Leur grande variété pourrait permettre aux populations d'assurer leur sécurité alimentaire et des revenus supplémentaires, en particulier les femmes et les enfants (Herzog 1992 et 1994, Mollet et al. 2000, Sonwa et al. 2001). En effet, l'alimentation de base des populations du « V Baoulé ", dans lequel s'inscrit la zone d'étude, est basée sur les féculents (igname et banane), les légumes (feuilles de fromager), les fruits consommés crus (avocat) et les boissons (vin de palme). Selon Herzog (1992) la valeur nutritionnelle de certaines espèces dont Elaeis guineensis (Arecaceae) et Ricinodendron heudelotii (Euphorbiaceae) connu localement sous le nom de Akpi, permettait aux populations de couvrir leurs besoins en énergie et en vitamines. Les espèces utilisées comme bois d'œuvre et plantes ornementales constituent une ressource non négligeable dans des agroforêts car elles sont utilisées par les paysans soit pour la construction, soit pour être commercialisées. Leur faible représentativité dans les plantations pourrait être attribuée soit à leur utilisation comme bois de chauffe, soit à une certaine incompatibilité avec la culture cacaoyère, soit enfin à leur surexploitation.

\section{Choix des espèces compagnes des cacaoyers : une recherche permanente de compromis}

Bien que lors de la création de la parcelle cacaoyère le paysan soit guidé par le besoin d'ombrage, la sélection des espèces résulte d'un compromis entre leurs utilisations domestiques et leur incidence sur la culture (Sonwa et al. 2007). Les analyses ont montré que ce choix des espèces varie en fonction des localités, des usages et de leur valeur économique. Les années d'apprentissage effectuées auprès des parents ont donc permis aux paysans d'acquérir des connaissances empiriques de l'incidence des espèces sur divers aspects de la cacaoculture. Ainsi, cinq catégories d'espèces ont pu être répertoriées par ces derniers.

Suivant les facteurs, certaines espèces ont pu être identifiées comme étant favorables à la culture cacaoyère par un accroissement de la production. Dans cette catégorie, on retrouve exclusivement les espèces forestières alimentaires, médicinales ou utilisées comme bois d'œuvre. Cependant, leur choix se fonde principalement sur une possible incidence sur la fertilité du sol, le type d'usage étant alors relégué au second plan. Il s'agit principalement du Fromager (Ceiba pentandra), de l'Iroko (Milicia sp.), du Kapokier ( Bombax buonopozense), de l'Acajou (Khaya grandifoliola) et du Tiama (Entandrophragma angolense). Cette perception paysanne est liée au fait que ces espèces pourraient participer au maintien de la fertilité des sols. En effet, elles ont un ombrage léger et perdent leurs feuilles pendant la saison sèche. Les paysans leur attribuent ainsi également une décomposition rapide des feuilles et des semences comme le soulignent Mollet et al. (2000). Le choix de ces espèces peut s'expliquer par le décalage de leur phénologie par rapport à celle du cacaoyer qui pourrait jouer en faveur d'un phénomène de facilitation par l'augmentation de la ressource disponible (Isaac et al. 2007, Dupraz \& Liagre 2008). Pour certains auteurs en revanche, la persistance de ces grands arbres serait liée au fait que les paysans n'arrivent pas à les abattre pendant les défrichements agricoles (Zapfack et al. 2002, Adou Yao \& N'Guessan 2006). En réalité, les paysans ont appris avec le temps 
les deux mécanismes mis en œuvre simultanément dans toutes les associations culturales : la compétition et la facilitation (Isaac et al. 2007, Dupraz \& Liagre 2008). La facilitation se traduit par une modification de l'environnement de la culture par l'arbre et vice versa. Ainsi, la ressource à partager peut soit augmenter et réduire la compétition (cas des espèces du groupe G2), soit diminuer et donc accroître la compétition selon la nature des espèces associées. Ce dernier cas s'observe à travers les espèces considérées non favorables (non compatibles) à la cacaoculture. La principale raison de leur rejet est qu'elles provoquent un assèchement du sol. Cette perception se fonde sur plusieurs caractéristiques dont les plus importantes sont le système racinaire et la surface foliaire (Bidzanga et al. 2009).

On peut citer Nesogordonia papaverifera (Sterculiaceae) dans les localités enquêtées. Leurs racines superficielles ne favoriseraient pas la pénétration de l'eau dans le sol après une forte pluie. Les feuilles de Cola gigantea (Sterculiaceae) à en croire les paysans, ne se décomposent pas rapidement. La majorité des espèces citées dans ce groupe appartient à la famille des Sterculiaceae. Le maintien de ces espèces pourrait s'expliquer par la nécessité d'un ombrage permanent dans la plantation, par la présence de certaines variétés culturales (Amelonado). En effet, les analyses ont montré que c'est dans la localité de Niamkey-Konankro où l'on cultive le plus l'Amelonado que se rencontrent ces types d'espèces «indésirables». Pour Gidoin (2013), cette incompatibilité pourrait se justifier par le fait que le cacaoyer fait également partie de la famille des Sterculiaceae dont certains genres (Cola spp.) sont des hôtes des mirides.

Le maintien de ces espèces indésirables se justifie donc essentiellement par leurs usages. Ce cas de figure s'observe dans les groupes G4 et G5 de la classification obtenue. En effet, les espèces du groupe G5 constituent d'excellents bois de chauffage: Albizia zygia (Mimosaceae) et Celtis zenkeri (Ulmaceae). Dans le groupe G4, c'est l'importance alimentaire ou commerciale des espèces qui favorise leur maintien. C'est le cas dans ce groupe de Elaeis guineensis (Arecaceae) ou Borassus aethiopium (Arecaceae) encore appelé rônier, dont les produits servent à l'autoconsommation et sont facilement commercialisables. Ainsi, leur importance quotidienne a de nombreuses fois été décrite (Mollet et al. 2000, Vroh et al. 2014). La dernière catégorie G1 est constituée d'espèces exotiques telles que Persea americana (Lauraceae), Mangifera indica (Anacardiceae) et Citrus spp. (Rutaceae). Leur incidence sur la production cacaoyère ne fait pas l'unanimité dans la population étudiée car certaines sont des hôtes de Loranthaceae. Koko et al. (2013) ont également observé une baisse de rendement lorsque ces espèces sont plantées trop près des cacaoyers. Néanmoins, elles participent à la diversification des produits alimentaires ou des sources de revenus du paysan (Zohoun et al. 2002). Leur introduction massive dans les plantations de cacaoyers de Côte d'Ivoire et au Cameroun répond à une stratégie d'adaptation des paysans suite à la crise cacaoyère des années 1980 (Bidzanga et al. 2009, Tano 2012).

Cette classification paysanne de l'incidence des espèces en particulier sur le fait qu'elles favoriseraient la productivité des cacaoyers ne rencontre pas souvent l'assentiment des structures de recherches et d'encadrement paysans (Asare 2005). En croisant notre liste à celle d'Asare (2005), on note que dans l'ensemble des espèces citées par les paysans, onze d'entre elles sont reconnues comme étant compatibles avec la culture cacaoyère, selon les structures de recherche. Il s'agit de Milicia excelsa (Iroko), Terminalia superba (Fraké), Citrus sinensis (Oranger), Elaeis guineensis (Palmier à huile), Cola nitida (Colatier), Mangifera indica (Manguier), Triplochiton scleroxylon (Samba), Alstonia boonei (Emien), Ricinodendron 
heudelotii (Akpi), Cocos nucifera (Cocotier) et Persea americana (Avocatier). Des recherches menées au Ghana ont montré que Citrus spp. et Elaeis guineensis (Palmier à huile) isolent les cacaoyers de l'infection du virus du swollen shoot. Certaines des espèces citées dans des études comme favorisant la production, sont considérées antagonistes des cacaoyers dans la sous-région (Asare 2005). Il s'agit de Ceiba pentandra (Fromager), considéré comme hôte des mirides et du virus du swollen shoot. Des recherches approfondies sur les espèces hôtes des maladies du cacaoyer devraient être conduites sur le long terme pour mieux comprendre le rôle de ces espèces dans la cacaoculture.

\section{Conclusion}

31 La présente étude met en exergue l'existence de systèmes agroforestiers dans les localités de la zone de contact forêt-savane du centre de la Côte d'Ivoire. La mise en place de ces systèmes et leur gestion sont sous-tendues par des pratiques, des savoirs et savoir-faire mis en œuvre depuis l'introduction du cacao dans cette région. Ces pratiques et savoirs sont transmis de génération en génération. La gestion de ces systèmes agroforestiers a conduit les paysans à y maintenir, préserver ou introduire diverses espèces locales ou exotiques. La diversité de ces plantes associées aux cacaoyers dépend donc des savoirs et savoir-faire accumulés pendant longtemps par le cultivateur et surtout en tenant compte des bénéfices que celles-ci peuvent leur procurer. Ainsi l'étude a permis de recenser 105 espèces végétales fournissant des services d'approvisionnement et permettant d'assurer une sécurité alimentaire et sanitaire aux populations locales.

En considérant les perceptions des incidences éventuelles, 65 espèces, sont reconnues par les paysans comme étant compatibles ou non avec la culture cacaoyère. Celles citées comme favorables par les populations locales sont généralement représentées par les grands arbres comme Milicia excelsa qui maintiendraient la fertilité des sols ou l'humidité dans le milieu. Les espèces vues comme n'ayant aucune incidence sur le cacaoyer, sont représentées par les espèces alimentaires. Les espèces non favorables, dont Nesogordonia papaverifera, sont perçues comme asséchant le sol ou retardant le développement des cacaoyers. Les mécanismes de ces antagonismes sont souvent difficiles à expliquer par les paysans. Des recherches approfondies prenant en compte les aspects physiques, chimiques et même d'autres facteurs biologiques indicateurs de la fertilité des sols seront, cependant, nécessaires pour affiner la validité des connaissances des cacaoculteurs de la zone d'étude, sur les incidences des espèces.

BIBLIOGRAPHIE

Adou Yao C.Y. \& N'Guessan E.K. 2006 - Diversité floristique spontanée des plantations de café et de cacao dans la forêt classée de Monogaga, Côte d'Ivoire. Schweizerische Zeitschrift für Forstwessen 157 (2) : 31-36. 
Adou Yao C.Y. 2010 - Pratiques paysannes, biodiversité en forêt de Monogaga, Côte d'Ivoire : impacts des pratiques de gestion des populations locales Wanne et Bakwe et de la Sodefor sur la forêt classée de Monogaga. Éditions Universitaires Européennes, 232 p.

Asare R. 2005 - Cocoa agrofrest in West Africa: a look at activities on preferred trees in farming systems. Hørsholm (Denmark) : Forest \& Landscape Denmark (FLD), 89 p. (Working paper ; 6).

Berkes F. 2012 - Sacred ecology, Third Edition. New York, USA, Routledge, 392 p.

Bidzanga N., Fotsing B., Agoume V., Birang M.A., Onguene A.N. \& Zapfack L. 2009 - Mycotrophie et connaissances paysannes des essences fertilitaires dans les agroforêts à base de cacaoyers du sud Cameroun. Cameroon Journal of Experimental Biology 5 (2) : 79-86.

Cardinale B.J., Duffy J.E., Gonzalez A., Hooper D.U., Perrings C., Venail P., Narwani A., Mace G.M., Tilman D., Wardle D.A., Kinzig A.P., Daily G.C., Loreau M., Grace J.B., Larigauderie A., Srivastava D.S. \& Naeem S. 2012 - Biodiversity loss and its impact on humanity. Nature 486 : 59-67.

Carrière S. 1999 - «Les orphelins de la forêt » : influence de l'agriculture itinérante sur brûlis des Ntumu et des pratiques agricoles associées sur la dynamique forestière du sud Cameroun. Thèse de Doctorat, Sciences et Techniques du Languedoc Montpellier II, Montpellier, 376 p.

Cerda R., Deheuvels O., Calvache D., Niehaus L., Saenz Y., Kent J., Vilchez S., Villota A., Martinez C. \& Somarriba E. 2014 - Contribution of cocoa agroforestry systems to family income and domestic consumption: looking towards intensification. Agroforestry Systems 88 (6) : 957-981.

Chauveau J.-P. 1979 - Les cadres socio-historiques de la production dans la région de Kokoumbo (pays baoulé, Côte d'Ivoire). Bondy (France), ORSTOM, 143 p. (Cahier ORSTOM sciences humaine ; 7).

Clough Y., Barkmann J., Juhrbandt J., Kessler M., Wanger T. C., Anshary A., Buchori D., Cicuzza D., Darras K., Putra D., Erasmi S., Pitopang R., Schmidt C., Schulze C.H., Seidel. D., Steffan-Dewenter I., Stenchly K., Vidal S., Weist M., Wielgoss A.C. \& Tscharntke T. 2011 - Combining high biodiversity with high yields in tropical agroforests. Proceedings of the National Academy of Sciences $108(20)$ : 8311-8316.

Deheuvels O., Avelino J, Somarriba E. \& Malezieux E. 2012 - Vegetation structure and productivity in cocoa-based agroforestry systems in Talamanca, Costa Rica. Agriculture, Ecosystems and Environment 149 : 181-188.

Dupraz C. \& Liagre F. 2008 - Agroforesterie : des arbres et des cultures. Paris, France Agricole, 415 p.

Foyer J. 2008 - Diversité naturelle et culturelle face aux défis des biotechnologies : enjeux et controverses au Mexique. Thèse de Doctorat, Université de la Sorbonne nouvelle-Paris III, 666 p.

Gidoin C. 2013 - Relations entre structure du peuplement végétal et bioagresseurs dans les agroforêts à cacaoyers. Application à trois bioagresseurs du cacaoyer : la moniliose au Costa Rica, la pourriture brune et les mirides au Cameroun. Thèse de Doctorat, Collège doctoral du Languedoc-Roussillon,

Montpellier, $2010 \mathrm{p}$.

Guillaumet J.-L. \& Adjanohoun E. 1971 - La végétation de la Côte d'Ivoire. In : Avenard J.M., Eldin E., Girard G., Sircoulon J, Touchebeuf P, Guillaumet J.L., Adjanohoun E. \& Perraud A. (Ed.), Le Milieu naturel de la Côte d'Ivoire. Paris, ORSTOM : 161- 263. (Mémoires ORSTOM ; 50).

Hernandez-Morcillo M., Hoberg J., Oteros-Rozas E., Plieninger T., Gomez-Baggethun E. \& ReyesGarcia V. 2014 - Traditional Ecological Knowledge in Europe Status Quo and Insights for the Environmental Policy Agenda. Environment 56 : 3-17. 
Herzog F.M. 1992 - Étude biochimique et nutritionnelle des plantes alimentaires sauvages dans le sud du V-Baoulé, Côte d'Ivoire. Thèse de doctorat, École Polytechnique Fédérale Zurich, Suisse, $134 \mathrm{p}$.

Herzog F.M. 1994 - Multipurpose shade trees in coffee and cocoa plantations in Côte d'Ivoire. Agroforestry systems 27 : 259-267.

Hiernaux P. 1975 - Étude phytoécologique du pays baoulé méridional (Côte d'Ivoire centrale). Thèse Doctorat Ingénieur, CNRS Montpellier, France, 206 p.

ICCO 2008 - Rapport annuel 2008/2009. London WC1A 1NU, United Kingdom, 42 p.

Isaac M.E., Timmer V.R. \& Quashie-Sam S.J. 2007 - Shade tree effects in an 8-year old cocoa agroforestry system: biomass and nutrient diagnosis of Theobroma cacao by vector analysis. Nutrient Cycling Agroecosystems 78 : 155-165.

Jose S. 2009 - Agroforestry for ecosystem services and environmental benefits: an overview. Agroforestry systems $76: 1-10$.

Kasisi R. \& Jacobs P. 2002 - Les stratégies et plans d'action pour la conservation de la diversité biologique : un défi culturel et scientifique. Ressources marines et traditions - Bulletin de la CPS 13 : 14-23.

Koko L.K., Snoeck D., Lekadou T.T. \& Assiri A.A. 2013 - Cacao-fruit tree intercropping effects on cocoa yield, plant vigour and light interception in Côte d'Ivoire. Agroforestry Systems 87 : 1043-1052.

Kpangui K.B., Kouamé D., Gone B.Z.B., Vroh B.T.A., Koffi B.J.C. \& Adou Yao C.Y. 2015 - Typology of cocoa-based agroforestry systems in a forest-savannah transition zone: case study of Kokoumbo (Centre, Côte d'Ivoire). International Journal of Agronomy and Agricultural Research (IJAAR) 6 (3) : 36-47.

Mollet M., Téré H. \& Herzog F. 2000 - Ligneux à usages multiples dans les systèmes agraires tropicaux: une étude de cas de Côte d'Ivoire. Schweizerische Zeitschrift für Forstwessen 151 (10) : 355-364.

Nabanoga K.G.N. 2005 - Transgressing boundaries: Gendered spaces, species, and indigenous forest management in Uganda. PhD Thesis, Wageningen University, Tropical Resource Management Papers 60, $227 \mathrm{p}$.

Perraud A. 1971 - Les sols. In : Avenard J.M., Eldin E., Girard G., Sircoulon J, Touchebeuf P, Guillaumet J.-L., Adjanohoun E. \& Perraud A. (Ed.), Le Milieu naturel de la Côte d'Ivoire. Paris, ORSTOM : 263-327. (Mémoires ORSTOM ; 50).

Rice R. \& Greenberg R. 2000 - Cacao cultivation and the conservation of biological diversity. Ambio 29 : 167-173.

Roué M., Battesti V., Césard N. \& Simenel R. 2015 - Ethnoecology of pollination and pollinators. Revue d'ethnoécologie [en ligne]) 7, mise en ligne le 01 juillet 2015, consulté le 10 août 2015. URL : http://ethnoecologie.revues.org/2229; DOI : 10.4000/ethnoecologie.2229

Ruf F.O. \& Zadi H. 1998 - Cocoa: From Deforestation to Reforestation. Conférence sur la production durable du cacao au Panama, 30 Mars au 21 avril 1998, 8 p.

Schroth G., da Fonseca G.A.B., Harvey C.A., Gascon C., Vasconcelos H.L. \& Izac A-M.N. 2004 - A groforestry and Biodiversity Conservation in Tropical Landscapes. Washington DC (USA), Island Press, $537 \mathrm{p}$.

Somarriba E. \& Beer J. 2011 - Productivity of Theobroma cacao agroforestry systems with timber or legume service shade trees. Agroforestry Systems 81 : 109-121. 
Sonwa D.J., Nkongmeneck B.A., Weise S.F., Tchatat M., Adesina A.A. \& Janssens M.J.J. 2007 - Diversity of plants in cocoa agroforests in the humid forest zone of Southern Cameroon. Biodiversity and Conservation $16: 2385-2400$.

Sonwa D.J., Weise S.F., Tchatat M., Nkongmeneck B.A., Adesina A.A., Ndoye O. \& Gockowski J., 2001 - Rôle des agroforêts à cacao dans la foresterie paysanne et communautaire au sud-Cameroun. Document RDFN, $\mathrm{n}^{\circ} 25 \mathrm{~g}$ (i), $12 \mathrm{p}$.

Takeuchi K. 2010 - Rebuilding the relationship between people and nature: the Satoyama Initiative. Ecological Research 25 : 891-897.

Tano M.A. 2012 - Crise cacaoyère et stratégies des producteurs de la sous-préfecture de Méadji au Sudouest ivoirien. Thèse de Doctorat, Université Toulouse le Mirail-Toulouse II, France, 263 p.

Vaast P. \& Somarriba E. 2014 - Trade-offs between crop intensification and ecosystem services: the role of agroforestry in cocoa cultivation. Agroforestry Systems 88 : 947-956.

Vroh B.T.A.; Ouattara D. \& Kpangui K.B. 2014 - Disponibilité des espèces végétales spontanées à usage traditionnel dans la localité d'Agbaou, Centre-ouest de la Côte d'Ivoire. Journal of Applied Biosciences 76 : 6386-6396.

Yao-Kouamé A. 2007 - Caractéristiques physiques des sols brunifiés dérivés des formations du complexe volcano-sédimentaire de Kanhankro (Toumodi) en moyenne Côte d'Ivoire. Rev. CAMESSérie A 05 : 76-86.

Zapfack L., Engwald S., Sonke B., Achoundong G. \& Madong B. A. 2002 - The impact of land conversion on plant biodiversity in the forest zone of Cameroon. Biodiversity and Conservation 11 : 2047-2061.

Zohoun G., Boya Y., Attolou M., Adjakidje V., Oudé P. \& Houndaye F. 2002 - L'utilisation des produits forestiers non ligneux (PFNL) dans le cadre de la gestion forestière durable au Bénin. Le Flamboyant $55: 13-18$.

\section{RÉSUMÉS}

La cacaoculture a été longtemps considérée comme l'une des principales causes de dégradation du couvert forestier dans le monde tropical. Cependant, de plus en plus, des études ont montré que les systèmes agroforestiers à base de cacaoyers renferment une plus grande diversité d'espèces d'ombrage que les plantations monoculturales. Outre les biens et services fournis par ces espèces, seules les perceptions des paysans locaux peuvent permettre le maintien et la réussite de ce système dans les zones de contacts forêt-savane, considérées comme peu favorables à la cacaoculture. La présente étude a permis d'analyser les pratiques agroforestières traditionnelles dans trois localités de Kokoumbo au centre de la Côte d'Ivoire. Les usages des espèces végétales présentes dans les plantations et les perceptions des populations de leurs incidences sur le cacaoyer, ont fait l'objet d'enquêtes auprès de 105 paysans. Les données qui en ont résulté ont été complétées par des inventaires botaniques dans des parcelles de $625 \mathrm{~m}^{2}$. Les résultats montrent que 65 espèces sont reconnues par les paysans comme étant compatibles ou non avec la culture cacaoyère. De grands arbres comme Milicia excelsa qui maintiendraient la fertilité des sols ou l'humidité dans le milieu, sont cités comme compatibles avec la culture des cacaoyers. La majorité des espèces alimentaires n'ont pas d'incidence connue sur le cacaoyer. Nesogordonia papaverifera, bien que fréquemment rencontrée dans les plantations, est connue comme asséchant le sol ou retardant le développement des cacaoyers. Les mécanismes de ces antagonismes sont souvent difficiles à expliquer par les paysans. Des recherches approfondies 
prenant en compte les aspects physiques, chimiques et même d'autres facteurs biologiques, indicateurs de la fertilité des sols seront nécessaires pour étayer les connaissances des cultivateurs locaux de cacao de la zone d'étude.

Cocoa production has long been considered as one of the main causes of forest cover degradation in the tropics. However, studies show more and more often that cocoa-based agroforestry systems host a relativily high diversity of shade species, compared to monoculture plantations. In addition to the goods and services provided by these species, only local farmers' perceptions could allow the maintenance and success of this cultivation system in forest-savanna transition zone, considered as unfavorable for cocoa cultivation. Our study analyses the traditional agroforestry practices in three localities of Kokoumbo in the Centre of Côte d'Ivoire. One hundred and five peasants have been surveyed and we have recorded their utilization of plant species in cocoa plantations and their perceptions of the repercssions on cocoa trees. The resulted data have been completed by botanical inventories in 105 plots of $625 \mathrm{~m}^{2}$ each. The results show that 65 species were recognized by farmers as being compatible or not with the cocoa cultivation. Large trees like Milicia excelsa that would maintain soil fertility or moisture in the environment, were marked favorable to cocoa. The majority of food species had no known effect on cocoa trees. Nesogordonia papaverifera, although commonly found in plantations, was known to dry soil or delay the development of cocoa trees. The mechanisms of these antagonisms were often difficult to explain by farmers. Extensive research taking into account the physical, chemical aspects and even other biological factors, indicators of soil fertility will be necessary to support the knowledge of local cocoa farmers in the study area.

INDEX

Index géographique : Côte d'Ivoire

Mots-clés : espèces d'ombrage, pratiques paysannes, connaissances des communautés locales, cacaoculture

Keywords : shade species, farming practices, local community knowledge, cocoa cultivation, Côte d'Ivoire

\section{AUTEURS}

\section{CONSTANT YVES ADOU YAO}

Maître de Conférences du Cames en Botanique tropicale \& Ethnobiologie

Laboratoire de Botanique, UFR Biosciences, Université Félix Houphouët-Boigny (UFHB), Côte

d'Ivoire, 22 BP 582 Abidjan 22

Centre Suisse de Recherches Scientifiques en Côte d'Ivoire

adouyaocy@gmail.com

\section{KOUASSI BRUNO KPANGUI}

Assistant en Botanique/Écologie végétale et SIG

Laboratoire de Botanique, UFR Environnement, Université Jean Lorougnon Guédé (UJLoG), Daloa, Côte d'Ivoire, BP 150 Daloa 


\section{BI TRA AIMÉ VROH}

Assistant en Botanique/Écologie végétale

Laboratoire de Botanique, UFR Biosciences, Université Félix Houphouët-Boigny (UFHB), Côte d'Ivoire, 22 BP 582 Abidjan 22

\section{DJAKALIA OUATTARA}

Maître de Conférences du Cames en Botanique/Ethnobotanique

Laboratoire de Botanique, UFR Biosciences, Université Félix Houphouët-Boigny (UFHB), Côte

d'Ivoire, 22 BP 582 Abidjan 22 